\title{
Gamificação no ensino de enfermagem: avaliação do impacto na aprendizagem
}

\author{
Gamification in nursing teaching: assessment of the impact on learning
}

Gamificación en la enfermería: evaluación del impacto en el aprendizaje

Recebido: 25/11/2021 | Revisado: 02/12/2021 | Aceito: 11/12/2021 | Publicado: 19/12/2021

Queila Samara dos Santos Farias ORCID: https://orcid.org/0000-0002-4686-4539 Universidade Tiradentes, Brasil E-mail: queilasamara20@gmail.com

Renata Santos da Silva

ORCID: https://orcid.org/0000-0001-5459-3444 Universidade Tiradentes, Brasil

E-mail: renatasilvasts@yahoo.com

Jéssica Mayara da Silva Araújo ORCID: https://orcid.org/0000-0003-0453-4416 Universidade Tiradentes, Brasil

E-mail: jessicamayara3357@gmail.com

Maria Mylena Gomes Santos ORCID: https://orcid.org/0000-0002-9555-8175 Universidade Tiradentes, Brasil E-mail: myly.myh@hotmail.com

Fernanda Dantas Barros

ORCID: https://orcid.org/0000-0001-9155-1665 Universidade Tiradentes, Brasil

E-mail: nanda-dantas@ hotmail.com

Manuela de Carvalho Vieira Martins

ORCID: https://orcid.org/0000-0003-1222-5955 Universidade Tiradentes, Brasil

E-mail: manuela.cvm@hotmail.com

Fernanda Costa Martins Gallotti

ORCID: https://orcid.org/0000-0002-9063-1273 Universidade Tiradentes, Brasil

E-mail: fernanda.gallotti@souunit.com.br

\begin{abstract}
Resumo
Este estudo tem como objetivo identificar as evidências disponíveis na literatura sobre a eficácia da gamificação como estratégia de ensino aprendizagem na formação do enfermeiro. Trata-se de uma revisão de escopo, desenvolvida de acordo com a metodologia do Instituto Joanna Briggs. Utilizou-se as bases de dados Cochrane, PubMed, Lilacs, Science Direct e ERIC. A estratégia de busca foi ajustada de acordo com os protocolos de cada base e os descritores para combinações foram definidos a partir do vocabulário controlado da Medical Subject Headings (MeSH). Foram utilizados documentos publicados nos últimos cinco anos, com amostra final composta por 16 artigos. Observou o predomínio da aplicação da gamificação de forma online, tendo dois estudos que trouxeram a utilização dos games de maneira presencial. Todos os estudos apontaram como positiva a utilização da gamificação em sala de aula. Evidencia também que a mesma pode ser entendida como um recurso adicional no trabalho do docente, trazendo uma aprendizagem significativa e um ambiente seguro. Há escassez de estudos relacionados a eficácia da gamificação na formação do enfermeiro, mas pode-se observar a extrema importância da inserção dos games como estratégia de ensino e aprendizagem, por ajudar o corpo discente no seu desenvolvimento intelectual e pessoal.
\end{abstract}

Palavras-chave: Aprendizagem; Educação baseada em competências; Educação em enfermagem.

\begin{abstract}
This study aims to identify the evidence available in the literature on the effectiveness of gamification as a teachinglearning strategy in nursing education. This is a scope review, developed according to the methodology of the Joanna Briggs Institute. The Cochrane, PubMed, Lilacs, Science Direct and ERIC databases were used. The search strategy was adjusted according to the protocols of each database and the descriptors for combinations were defined from the controlled vocabulary of the Medical Subject Headings (MeSH). Documents published in the last five years were used, with a final sample of 16 articles. It observed the predominance of the application of gamification online, having two studies that brought the use of games in person. All studies pointed out as positive the use of gamification in the classroom. It also shows that it can be understood as an additional resource in the teacher's work, bringing significant learning and a safe environment. There is a scarcity of studies related to the effectiveness of gamification in nursing
\end{abstract}


education, but one can observe the extreme importance of inserting games as a teaching and learning strategy, as they help the student body in their intellectual and personal development.

Keywords: Learning; Competency-based education; Nursing education.

\section{Resumen}

Este estudio tiene como objetivo identificar la evidencia disponible en la literatura sobre la efectividad de la gamificación como estrategia de enseñanza-aprendizaje en la educación en enfermería. Se trata de una revisión del alcance, desarrollada según la metodología del Instituto Joanna Briggs. Se utilizaron las bases de datos Cochrane, PubMed, Lilacs, Science Direct y ERIC. La estrategia de búsqueda se ajustó según los protocolos de cada base de datos y los descriptores para las combinaciones se definieron a partir del vocabulario controlado de los Medical Subject Headings (MeSH). Se utilizaron documentos publicados en los últimos cinco años, con una muestra final de 16 artículos. Observó el predominio de la aplicación de gamificación online, habiendo dos estudios que trajeron el uso de juegos de forma presencial. Todos los estudios señalaron como positivo el uso de la gamificación en el aula. También muestra que puede entenderse como un recurso adicional en el trabajo del docente, aportando un aprendizaje significativo y un entorno seguro. Existe una escasez de estudios relacionados con la efectividad de la gamificación en la formación en enfermería, pero se puede observar la extrema importancia de la inserción de juegos como estrategia de enseñanza y aprendizaje, ya que ayudan al alumnado en su desarrollo intelectual y personal.

Palabras clave: Aprendizaje; Educación basada en competencias; Educación en enfermería.

\section{Introdução}

A busca por estratégias na educação está mais alinhada ao perfil dos jovens e adultos que ingressam no ensino profissional, impulsionando pesquisas científicas em direção a concepções mais contemporâneas dos processos de ensinoaprendizagem (Giordano et al., 2021). A gamificação surge como uma estratégia de ensino que utiliza aplicações de elementos do design de jogos em contextos não-lúdicos, envolvendo estilo, pensamento e mecânica para a resolução de problemas reais (Toledo \& Kahler, 2019).

As técnicas de gamificação geralmente incluem níveis, sistemas de pontuação e emblemas para indicar progresso do jogador nas tarefas que lhes foram designadas e interação com os demais participantes. Somado a isso, cria uma esfera única com aspectos raros, como desafios, metas, feedback, recompensas, sistema de pontos, competição, colaboração e ranking (Michels et al., 2019).

Nesse contexto, a educação deve acompanhar a evolução da sociedade e procurar novas descobertas e novas ideias que possibilitem a construção de um ambiente propício para o aprendizado, provocando os indivíduos para o saber e, assim, ajudálos no seu desenvolvimento intelectual e pessoal (Orlandi et al., 2018).

As Diretrizes Curriculares Nacionais (DCNs) para o curso de Enfermagem destacam a necessidade de utilizar Metodologias Ativas (MA) no ensino-aprendizagem, incorporação de atividades complementares que contemplem as necessidades e expectativas individuais na formação dos estudantes. Esses fundamentos devem proporcionar um perfil profissional ético-legal, com responsabilidade social para atuar com qualidade, efetividade e resolubilidade, como também criticidade, autonomia e competência (Ministério da Saúde, 2018). Diante disso, é notório que as MA apresentam potencial como estratégia pedagógica no ensino em saúde (Fini, 2018).

A utilização de jogos e seus elementos de gamificação em estratégias pedagógicas tem sido adotada com o intuito de envolver e motivar os estudantes, favorecendo a aprendizagem. Mostrou-se também que a gamificação na educação de enfermagem proporciona o aprendizado com qualidade e que estimula as habilidades de pensamento crítico em um ambiente motivado e livre de estresse (Nascimento \& Feitosa, 2020; Cruz, 2017; Mcenroe-Petitte \& Farris, 2020).

Souza, Silva e Silva (2018) apontam que a utilização dessas metodologias possui grande relevância na formação do profissional de enfermagem por aprimorar as competências necessárias na integração entre a teoria e a prática. Assim, possibilitará uma maior aproximação do estudante com o processo saúde-doença do cliente e potencializará a interação entre pesquisa, ensino e assistência (Silva et al., 2020; Conceicao \& Moraes, 2018). 
Sob essa perspectiva, as Instituições de Ensino Superior (IES) vêm buscando promover mudanças no processo de ensino-aprendizagem de seus cursos, vislumbrando a formação de profissionais de saúde capazes de desenvolver competências e habilidades previstas nas DCN's (Oliveira; Rodrigues; Freitas, 2018).

Diante desses pressupostos, é pertinente estudar como a gamificação contribui como estratégia de ensino-aprendizagem na graduação de enfermagem, pois no entendimento educacional, o potencial da gamificação é alto e relevante. Somado a isso, a escassez de estudos científicos atualizados nos bancos de dados voltados a essa temática justifica a construção deste artigo como forma de divulgar essa metodologia entre os docentes e discentes da enfermagem.

Após contextualização do contéudo, algumas indagações acerca da proposta do estudo nos rementem a refletir a seguinte questão norteadora: qual(is) a(s) evidência(s) acerca da eficácia da gamificação como estratégia de aprendizagem na formação do enfermeiro? Nessa perspectiva, esta pesquisa tem como objetivo identificar as evidências disponíveis na literatura sobre a eficácia da gamificação como estratégia de ensino aprendizagem na formação do enfermeiro.

\section{Metodologia}

Trata-se de uma revisão de escopo, desenvolvida de acordo com a metodologia do Instituto Joanna Briggs. Optou-se pela realização de um estudo do tipo scoping review, por estar sendo amplamente utilizado na área das ciências da saúde, com o propósito de sintetizar e de dissipar os resultados dos estudos a respeito de um conteúdo. O objetivo da análise de escopo é mapear, através de um método rigoroso e transparente, o estado da arte em uma área temática, almejando fornecer uma visão descritiva dos estudos revisados, sem avaliá-los criticamente ou sumarizar evidências de diferentes investigações, como sucede em uma revisão sistemática (Tricco et al., 2018; Arksey, 2005).

Esta pesquisa foi norteada pelo protocolo Preferred Reporting Items for Systematic Reviews and Meta-Analyses extension for scoping review (PRISMA-ScR) (Tricco, 2018).

\section{Critérios de elegibilidade e estratégia de busca}

Para estruturação das estratégias de busca utilizou-se o Método PCC (P: População, C: Conceito, C: Contexto), para melhor formulação da pergunta norteadora desta pesquisa. Dessa forma, a pergunta de investigação da Scoping Review foi: Qual(is) a(s) evidência(s) acerca da eficácia da gamificação como estratégia de aprendizagem na formação do enfermeiro?

A busca foi realizada nas bases de dados: Cochrane, PubMed, Latin American and Caribbean Center on Health Sciences Information (Lilacs), Science Direct e Education Resources Information Center (ERIC). A estratégia de busca foi ajustada de acordo com os protocolos de cada base e os descritores para combinações foram definidos a partir do vocabulário controlado da Medical Subject Headings (MeSH), com o uso dos operadores Booleanos "AND" e "OR", sendo utilizados: estudantes de enfermagem; students nursing; aprendizagem baseada em problemas; Experimental game; game; Educação em enfermagem; Education; Nursing; "Learning" OR "Methods, Teaching".

De modo a explorar a estratégia de busca, termos não padronizados foram adicionados. A estratégia completa por base de dados incluída na pesquisa, foi descrita no Quadro 1. 
Quadro 1 - Expressões de busca utilizadas nas bases de dados incluídas no estudo. Recorte temporal dos últimos cinco anos (2016-2021).

\begin{tabular}{|c|c|}
\hline Base de dados & Estratégia de busca \\
\hline COCHRANE & $\begin{array}{l}\text { "Experimental game" OR "Game, Video" OR "Game, computer" AND } \\
\text { "Education, Nursing" OR "Student, Nursing" AND "Learning"" with } \\
\text { Publication Year from } 2016 \text { to 2021, in Trials }\end{array}$ \\
\hline PUBMED & $\begin{array}{l}\text { "Game, Experimental" OR "game" OR "Game, Video" OR "Game, } \\
\text { computer" AND "Education, Nursing" OR "Nursing Educations" OR } \\
\text { "Student, Nursing" OR "Education, Nursing, Diploma Programs" AND } \\
\text { "Learning" OR "Methods, Teaching" Abstract, in the last } 5 \text { years }\end{array}$ \\
\hline $\begin{array}{l}\text { Literatura Latino-Americana e do Caribe } \\
\text { em Ciências da Saúde (LILACS) }\end{array}$ & $\begin{array}{l}\text { ("Estudantes de Enfermagem" OR "Educação em enfermagem" AND } \\
\text { "Jogos de Vídeo" OR "Jogos experimentais" AND "aprendizagem") } \\
\text { ("Nursing Students" OR "Nursing Education" AND "Video Games" OR } \\
\text { "Experimental Games" AND "Learning") ("Estudiantes de enfermería" } \\
\text { OR "Educación en enfermería" AND "Videojuegos" OR "Juegos } \\
\text { experimentales" AND "Aprendizaje") }\end{array}$ \\
\hline $\begin{array}{l}\text { Education Resources Information Center } \\
\text { (ERIC) }\end{array}$ & $\begin{array}{l}\text { "Game, Experimental" OR "game" OR "Game, Video" OR "Game, } \\
\text { computer" AND "Education, Nursing" OR "Nursing Educations" OR } \\
\text { "Student, Nursing" OR "Education, Nursing, Diploma Programs" AND } \\
\text { "Learning" OR OR "Methods, Teaching" }\end{array}$ \\
\hline Science Direct & $\begin{array}{l}\text { "Game, Experimental" OR "Game, Video" OR "Game, computer" AND } \\
\text { "Education, Nursing" OR "Student, Nursing" OR "Education, Nursing, } \\
\text { Diploma Programs" AND "Learning" OR "Methods, Teaching" }\end{array}$ \\
\hline
\end{tabular}

Fonte: Autores.

Os critérios de inclusão aplicados nesta pesquisa foram: estudos originais, publicados no período de 2016 até agosto de 2021, em português, espanhol ou inglês, que apresentassem como objeto de investigação a aplicação de jogos como estratégia de ensino aprendizagem nos cursos de enfermagem. Foram excluídos os estudos: não disponíveis na íntegra, cuja solicitação de arquivo não foi respondida pelo autor e publicações em congressos. A busca dos estudos foi realizada em setembro de 2021.

\section{Seleção dos artigos e coleta dos dados}

Os títulos dos estudos foram organizados em planilha do software Microsoft Excel by Office 2019 e com uso do gerenciador de referências Mendeley, foi realizada a identificação e exclusão dos arquivos em duplicatas, seguida da exclusão de trabalhos duplicados identificados manualmente.

A seleção mediante critérios de elegibilidade foi realizada por dois revisores (Q.S.S.F e R.S.S), de forma independente. Os estudos que geraram alguma discordância entre os revisores quanto à inclusão foram discutidos com um terceiro revisor (F.C.M.G).

Após triagem por título, resumo e leitura na integra, os artigos selecionados foram analisados pelos revisores e extraída as seguintes informações: autores, ano de publicação, país de realização do estudo, tipo de estudo, número de estudantes inseridos no estudo, objetivo da pesquisa, foco do game, características da narração e os principais resultados encontrados nas pesquisas.

Em casos de divergência na leitura do texto completo, os revisores, em reuniões, buscavam um consenso. Caso a pesquisa fosse considerada inelegível a razão primária da exclusão foi registrada para composição do fluxo de seleção dos artigos.

\section{Análise dos dados}

Os dados extraídos dos estudos incluídos na Scoping Review foram apresentados por meio de análise descritiva das principais características dos estudos, visando apontar a relação entre a gamificação e a retenção da aprendizagem. 


\section{Resultados e Discussão}

Diante da triagem inicial realizada nas bases de dados utilizando a estratégia de busca, foram encontrados 1.684 documentos publicados nos últimos cinco anos. Após a coleta dos documentos, procedeu-se a tabulação dos dados com o auxílio do software Microsoft Excel. Excluiu-se 60 publicações indexadas de forma simultânea em duas ou mais bases de dados. Com a finalidade de cumprir com o objetivo proposto, realizou-se a leitura criteriosa dos títulos e dos resumos que resultaram na seleção de 27 artigos para leitura na íntegra. A amostra final desse estudo foi composta por 16 artigos (Figura 1).

Figura 1 - Fluxograma da seleção dos estudos que compõem a pesquisa de acordo com o PRISMA- ScR Diagram flow, 2021.

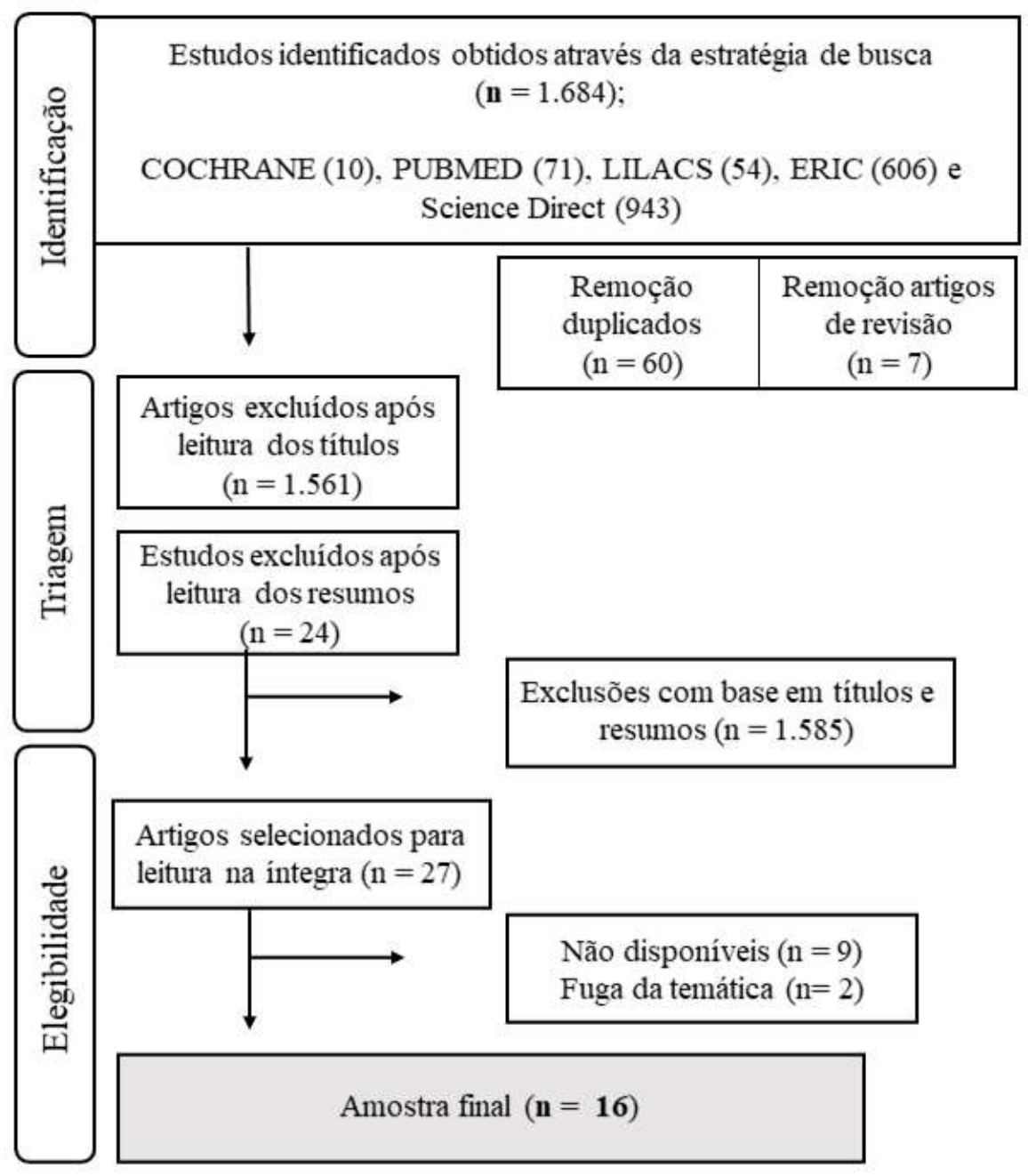

Fonte: Dados da pesquisa (2021).

Considerando o recorte temporal de 2016 a 2021, observou-se aumento no número de publicações no ano de 2019 com $31 \%$ artigos, seguido por 2018, com um total de $19 \%$. Nota-se a carência de pesquisas nos anos de 2020 e 2021 , cada um obteve o total de dois artigos publicados (12,5\%). Em relação ao tipo de estudo dessas pesquisas, destacou-se o estudo descritivo $(31,25 \%)$, seguido do ensaio clínico randomizado $(18,75 \%)$.

Nota-se através do Gráfico 1 que a aplicabilidade da gamificação está presente na maioria dos continentes, com um total de dez países diferentes com estudos publicados, a Singapura e Espanha tiveram um predomínio de artigos publicados acerca da temática, somando $(18,75 \%)$ cada, seguida do Canadá e Inglaterra com dois artigos, totalizando cada um (12,5\%). O Brasil totalizou apenas um estudo publicado (6,25\%). Verifica-se, então, a escassez e a necessidade de mais estudos na América Latina quando comparado ao quantitativo de pesquisas de outros continentes. 
Gráfico 1 - Número de artigos por país.

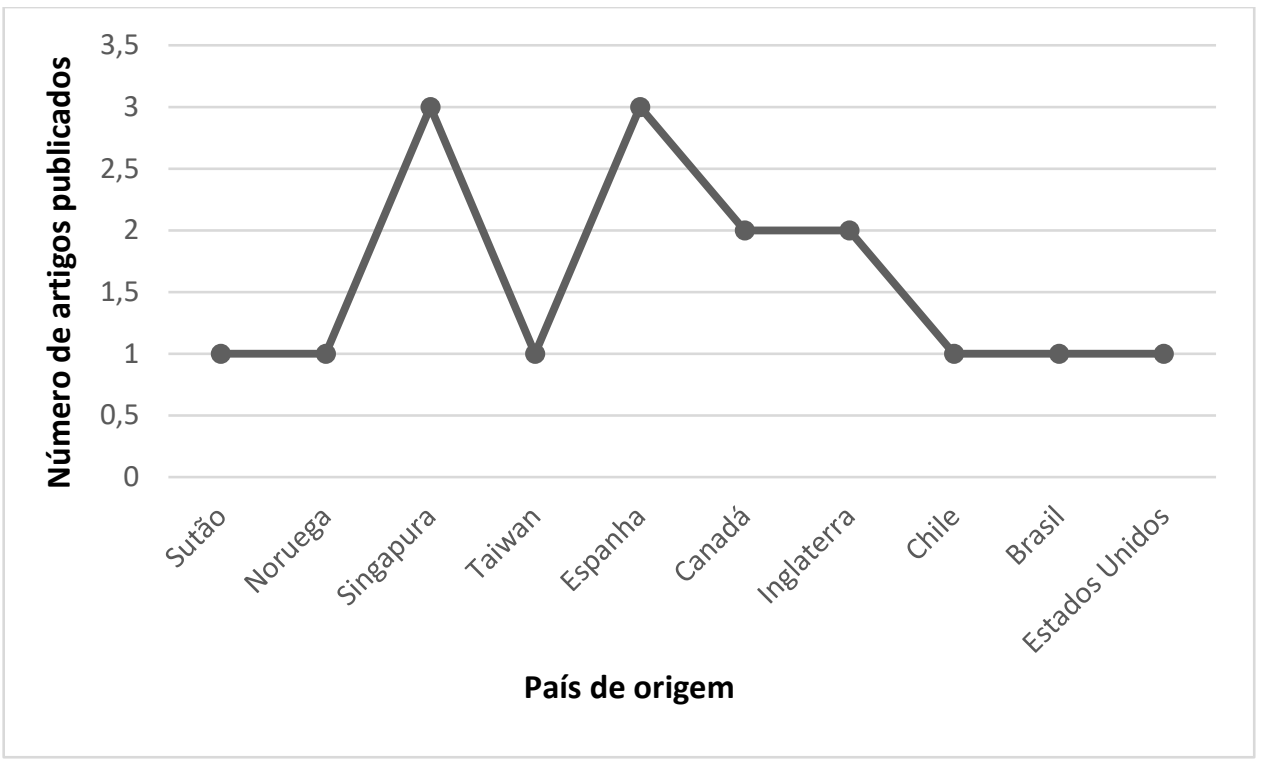

Fonte: Autoria própria (2021).

No que se observou na leitura na íntegra dos artigos, 18 publicações não atendiam ao objetivo proposto da presente pesquisa. Os motivos que levaram a exclusão incluíam indisponibilidade na íntegra, não se enquadrar na temática e ser revisão de estudo. O detalhamento de cada estudo encontra-se disponível na Tabela 1.

Tabela 1 - Caracterização dos estudos excluídos após leitura na íntegra, 2021.

\begin{tabular}{|c|c|c|}
\hline Autores, ano & $\begin{array}{c}\text { Título } \\
\end{array}$ & Motivo da exclusão \\
\hline CHING-HSING et al., 2018. & $\begin{array}{l}\text { Application of Team-Based Games in the Classroom: Teaching Medical } \\
\text { Terminology as an Example. }\end{array}$ & Indisponível na íntegra. \\
\hline ENGLUND et al., 2021. & $\begin{array}{l}\text { Bridging the Gap: Use of Educational Gaming to Teach Acid-Base } \\
\text { Imbalance Interpretation in Nursing Students. }\end{array}$ & Indisponível na íntegra. \\
\hline DENGHUI et al., 2021. & 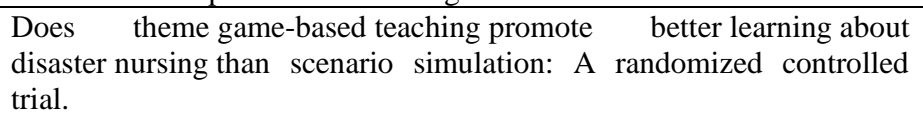 & Indisponível na íntegra. \\
\hline SARA et al., 2020. & $\begin{array}{l}\text { Game Elements and Instruments for Assessing Nursing Students' } \\
\text { Experiences in Learning Clinical Reasoning by Using Simulation } \\
\text { Games: An Integrative Review }\end{array}$ & Artigo de revisão. \\
\hline MEAGAN et al., 2018. & Gamification of Nursing Education With Digital Badges. & Artigo de revisão. \\
\hline REED, 2020. & Gaming in Nursing Education: Recent Trends and Future Paths. & Indisponível na íntegra. \\
\hline GARRISON et al., 2021. & Interactive Learning for Nurses Through Gamification. & Indisponível na íntegra. \\
\hline ANN; HARRIETT, 2017. & $\begin{array}{l}\text { Educationally Game-Based Learning Encourages Learners to Be } \\
\text { Actively Engaged in Their Own Learning }\end{array}$ & Artigo de revisão. \\
\hline ÇEKER, OZDAMLI, 2017 & What "Gamification" Is and What It's Not & Artigo de revisão. \\
\hline AN, 2020 & Designing Effective Gamified Learning Experiences & $\begin{array}{l}\text { Não se encaixa na } \\
\text { temática. }\end{array}$ \\
\hline ARNAB, CLARKE, 2015 & $\begin{array}{l}\text { Towards a Trans-Disciplinary Methodology for a Game-Based } \\
\text { Intervention Development Process }\end{array}$ & $\begin{array}{l}\text { Não se encaixa na } \\
\text { temática. }\end{array}$ \\
\hline URQUIZA et al., 2019 & $\begin{array}{l}\text { The impact on nursing students' opinions and motivation of using a } \\
\text { "Nursing Escape Room" as a teaching game: A descriptive study }\end{array}$ & Indisponível na íntegra. \\
\hline DO NASCIMENTO et al., 2021. & $\begin{array}{l}\text { Effectiveness of the serious game for learning in nursing: systematic } \\
\text { review }\end{array}$ & Artigo de revisão. \\
\hline BESSE et al., 2020. & The Simulation Games: Bringin' It to the Bedside & Indisponível na íntegra. \\
\hline GALLEGOS et al., 2017. & $\begin{array}{l}\text { The use of a game-based learning platform to engage nursing students: } \\
\text { A descriptive, qualitative study. }\end{array}$ & Indisponível na íntegra. \\
\hline YOUNG, 2018. & Using a Role-Play Simulation Game to Promote Systems Thinking & Indisponível na íntegra. \\
\hline BRULL et al., 2017. & $\begin{array}{l}\text { Using Gamification to Improve Productivity and Increase Knowledge } \\
\text { Retention During Orientation. }\end{array}$ & Artigo de revisão. \\
\hline ANN L, 2018. & Using Game-Based Virtual Reality with Haptics for Skill Acquisition & Artigo de revisão. \\
\hline
\end{tabular}


Na Tabela 2 encontram-se descritas as principais características dos artigos selecionados que compuseram as amostras utilizadas na pesquisa: referência, número de participantes, características do jogo, foco no game, programa de formação que enfatizam a eficácia da gamificação como estratégia de aprendizagem na formação do enfermeiro.

Quanto ao total de número de participantes nos estudos, como mostra na Tabela 2, houve uma variação de 5 a 560 participantes, com um total de 1.913 estudantes de enfermagem e enfermeiros. Das pesquisas analisadas, nove artigos envolveram alunos graduandos no curso de enfermagem, seis de cursos de capacitação para enfermeiros e um do programa de bacharelado em enfermagem.

Também é possível observar que o predomínio da aplicação da gamificação foi de forma online (87,5\%), tendo apenas dois estudos $(12,5 \%)$ que trouxeram a utilização dos games de maneira presencial. 
Tabela 2 - Características e principais resultados dos artigos incluídos neste estudo.

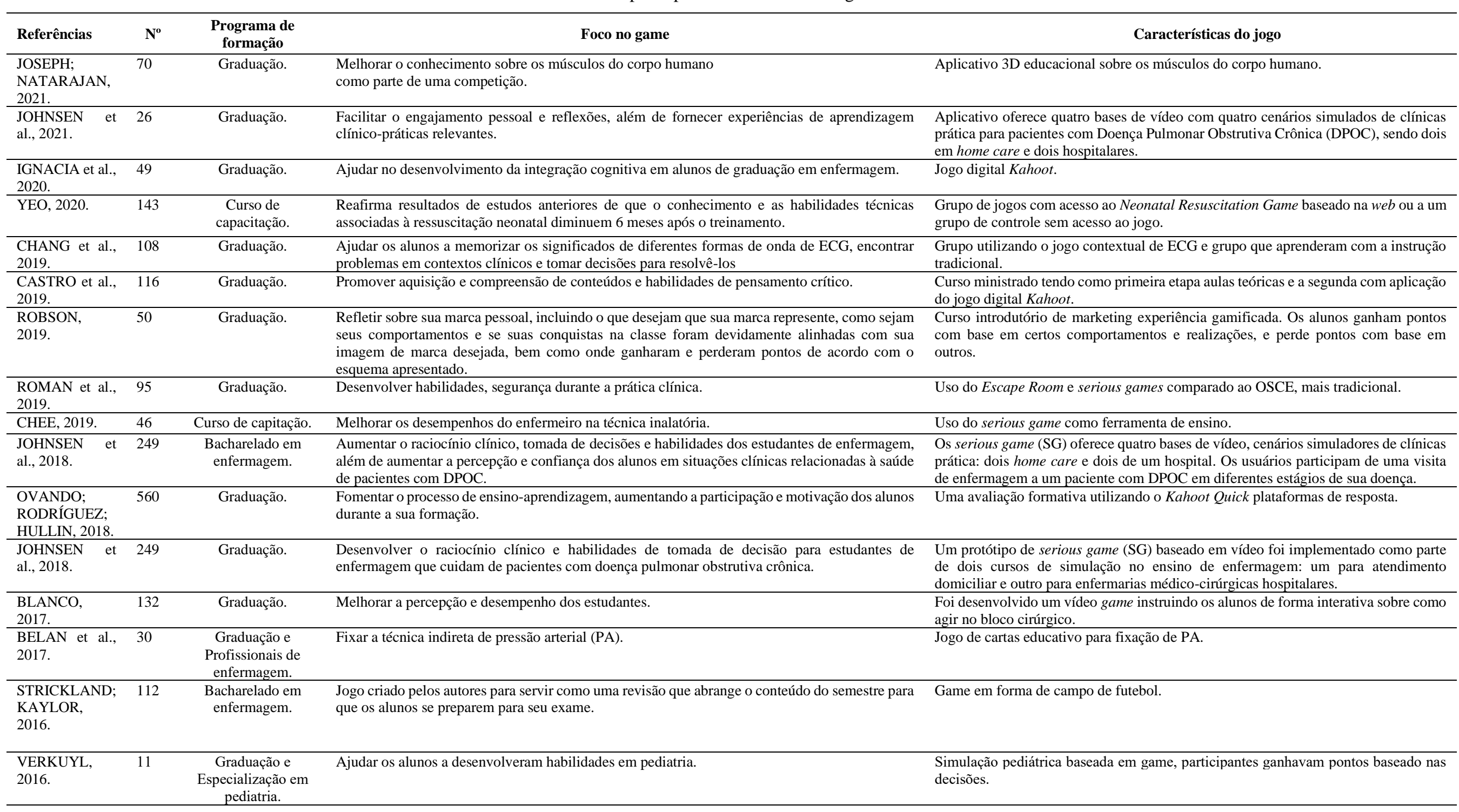

$\mathrm{N}^{\mathrm{o}}$ - Número de participantes envolvidos na pesquisa. Fonte: Autoria própria (2021). 
As pesquisas avaliaram os benefícios da gamificação na prática e validaram que pode melhorar a habilidade de trabalho em equipe, aumento da confiança frente ao paciente, raciocínio clínico, alto grau de satisfação com o jogo e com o conteúdo lecionado na dinâmica (Johnsen et al., 2018; Robson, 2019; Ovando, Rodríguez, Hullin, 2018; Verkuyl, 2016; Chee, 2019), aumento no número de aprovação (Yeo, 2020; Robson, 2019; Belan et al., 2017), ambiente descontraído (Blanco, 2017; Stricklando; Kaylor, 2016), motivação dos alunos (Chang et al., 2019). Apenas o estudo realizado por Ignacia e colaboradores (2020) discorda da utilização da gamificação como totalmente benéfica para os discentes.

Ao analisar os principais resultados dos artigos incluídos, pode-se observar a concordância de ideias sobre a aplicação dos jogos em sala de aula na maioria dos autores. Esses dados encontram-se dispostos na Tabela 3.

Tabela 3 - Principais resultados da pesquisa.

\section{Referências}

JOSEPH; NATARAJAN, 2021.

JOHNSEN et al., 2021.

IGNACIA et al., 2020.

YEO, 2020.

CHANG et al., 2019.

CASTRO et al., 2019.

ROBSON, 2019.

ROMAN et al., 2019.

ROMAN et al., 2019.

CHEE, 2019.

\section{Resultados}

Melhora de experiência de aprendizagem e aumento do interesse no curso.

Aumento do engajamento pessoal, ambiente de aprendizagem seguro e estruturado; Em relação aos diferentes temas, os alunos expressaram que as desvantagens de uma simulação foram neutralizadas na outra.

Promove o pensamento crítico e o raciocínio como também, é capaz de identificar suas lacunas de aprendizagem e, posteriormente, resolvê-las;

O uso de jogos em sala de aula era estressante, pois eles se preocupavam em ficar para trás dos seus pares.

85\% dos participantes do grupo treinados obtiveram aprovação de $80 \%$ nos testes em comparação com o grupo controle, $23 \%$, sugerindo deterioração mais rápida nas habilidades do que no conhecimento.

Melhora a motivação interna e extrínseca dos alunos;

O grupo experimental teve melhores atitudes de aprendizagem do que o grupo de controle, superando a tendência crítica nos pensamentos.

A taxa de acertos no jogo educacional foi maior que 50\% para todas as questões; Ferramenta foi de fácil utilização, avaliação positiva à aquisição e compreensão do conteúdo, bem como a interação professor-aluno;

59\% dos alunos da classe alcançaram atendimento perfeito. Apenas 3\% da classe recebeu deduções pelo uso do telefone nas aulas.

Desenvolve um ambiente mais descontraído que diminui os níveis de estresse, frustração do aluno durante a avaliação e promove o desenvolvimento de outras habilidades também exigidas na profissão.

Desenvolve um ambiente mais descontraído que diminui os níveis de estresse, frustração do aluno durante a avaliação e promove o desenvolvimento de outras habilidades também exigidas na profissão.

Os escores médios de autoeficácia pós-teste para o grupo experimental melhoraram após a intervenção e são mais altos em comparação com os escores médios pós-teste do grupo de controle. 
JOHNSEN et al., 2018.

OVANDO; RODRÍGUEZ; HULLIN, 2018.

JOHNSEN et al., 2018.

BLANCO, 2017.

BELAN et al., 2017.

STRICKLAND; KAYLOR, 2016.

VERKUYL, 2016.
Não foram encontradas diferenças significativas nas percepções do valor educacional entre estudantes de enfermagem com experiência anterior em saúde e aqueles sem nenhuma;

Os alunos dos cursos de simulação médico-cirúrgica e de assistência domiciliar sugeriram que mais SGs baseados em vídeo deveriam ser desenvolvidos e usados na educação de enfermagem, pois desenvolve o raciocínio clínico e habilidades de tomada de decisão.

Avaliaram a gamificação como motivacional e que melhorou a aprendizagem.

Os alunos sugeriram que mais SGs deveriam ser utilizados na educação de enfermagem, pois desenvolve o raciocínio clínico e habilidades de tomada de decisão.

Demonstraram menos medo de cometer erros; maior conhecimento percebido sobre como se comportar e o que podiam ou não fazer enquanto estivesse na sala de cirurgia e mostraram uma maior atitude colaborativa com pacientes e equipe.

93,3\% de acertos após aplicação do jogo;

Antes da aplicação do jogo, o quantitativo de participantes totalmente satisfeitos era $0 \%$, chegando a $13,3 \%$ no pós-teste.

Feedback positivo e promoção de um ambiente de ensino centrado no aluno, estimulando o interesse e a motivação dos alunos para aprender.

Alto grau de satisfação com o jogo. Os alunos relataram que aprenderam sobre cuidados pediátricos e estavam ansiosos para continuar jogando.

Fonte: Dados da pesquisa (2021).

Considerando a relação da eficácia da gamificação como estratégia de aprendizagem na formação do enfermeiro, os estudos que preencheram os critérios de inclusão foram limitados. Embora seja crescente a empregabilidade da gamificação em sala de aula, os anos de 2020 e 2021 apresentaram um decréscimo se comparado a 2019, ano com maior número de publicações e 2018, segundo ano com maior número.

As pesquisas incluídas no estudo apresentaram os benefícios e desvantagem da gamificação em sala de aula e nota-se que o predomínio dessa MA se dá nos países de primeiro mundo, como Singapura, Espanha, Estados Unidos, Noruega, Canadá e Taiwan. Com destaque a Singapura e Espanha que juntos totalizaram 37,5\% de publicações.

Sobre os benefícios da gamificação, os estudos mostram que os jogos em sala de aula aumentaram os interesses dos alunos com a temática utilizada, os estudantes se mostraram mais confiantes ao lidar com os pacientes, promoveu o pensamento clínico e reflexivo, maior habilidade nas tomadas de decisão e trabalho em equipe (Johnsen et al., 2018; Chee, 2019; Stricland; Kaylor, 2016; Verkuyl, 2016). Assim, pode-se colocar a lógica e metodologias dos jogos para tornar os conteúdos menos complexos e de fácil entendimento. Isso facilitará o processo de aprendizagem, incentivará os alunos a pensar e resolver problemas.

Somado a esses benefícios, pesquisas realizadas por Yeo (2020) e Robson (2019) apontaram acréscimo significativo no número de aprovação dos estudantes após aplicação da gamificação em sala de aula. O quantitativo de acertos nas questões após o uso do game aumentou 50\% no primeiro estudo e obteve 93,3\% de acertos em todas as questões no segundo, comprovando a eficácia do uso dos jogos no ensino superior (Castro et al., 2019; Belan et al., 2017). Corroborando com esses autores, os resultados apontados nos estudos de Chang e associados (2019) e Ovando et al. (2018), comprovam que os alunos se sentem mais motivados por aprender utilizando dinâmicas, favorecendo a atenção e aprendizagem.

Ao analisar as DCNs para cursos de enfermagem e suas competências na formação do enfermeiro, sustenta-se a importância de o profissional apresentar competências tecnológicas e habilidades em informática na sua prática cotidiana, 
considerando que a tecnologia tende a estar cada vez mais inserida aos processos de trabalho na saúde. Embora se reconheça os desafios na superação de dificuldades inerentes aos cenários de inovação, a inserção de metodologias ativas nos processos de ensino-aprendizagem, em programas de educação permanente e nas capacitações em serviço é considerada uma das possibilidades para ampliar esforços diante da rigidez dos métodos tradicionais encontrados no ensino da enfermagem (Vieira et al., 2016).

Nota-se que a utilização dos games tanto no ensino presencial, quanto no ensino remoto obteve uma interação satisfatória pelos alunos. As pesquisas indicam que os estudantes estavam motivados para participar das atividades propostas, e este tipo de metodologia deveria ser mais utilizado no ensino da enfermagem (Roman et al., 2019).

A pesquisa de Araújo e colaboradores (2016) traz que a gamificação utiliza elementos de jogos em contextos de não jogo, para criar uma prática diferenciada que respeite às necessidades e motivações humanas, como é visto nos resultados dos artigos, onde os estudantes retratam que avaliaram a gamificação como motivacional e que melhorou a aprendizagem. Diversas iniciativas de aplicação e de análise da gamificação no ensino podem ser reconhecidas no contexto brasileiro, fazendo-se possível afirmar que se trata de uma estratégia inovadora a qual tem conquistado espaço no âmbito educacional, o que explica a ideia de adequá-la ao ensino de enfermagem (Tenório et al., 2016).

$\mathrm{O}$ ambiente seguro para o aprendizado gerado pelo serious game dá-se pela possibilidade que o aluno tem em realizar diversas vezes o mesmo procedimento, permitindo erros para o aprimoramento das técnicas, uma vez que, a apresentação virtual não expõe o paciente ou prejudica a sua segurança (Silveira \& Cogo, 2017).

Como mencionado nos estudos realizados na Espanha e Estados Unidos em 2017 e 2016, respectivamente, apontam que os alunos demonstraram menos medo de cometer erros, maior conhecimento percebido sobre como se comportar frente as situações, além de mostrar maior atitude colaborativa com pacientes/equipe e promover um ambiente de ensino centrado no aluno, estimulando seu interesse e motivação para aprender (Blanco, 2017; Strickland \& Kaylor, 2016). Em contrapartida, a pesquisa realizada em Singapura no ano 2020 mostra que os discentes sinalizaram o uso de jogos em sala de aula estressante (Ignacia et al., 2020).

O uso do serious game é uma forte tendência à medida que o ensino online passa a ser enxergado como um complemento ao aprendizado presencial, dado que a sua facilidade de acesso, flexibilidade, bem como integração entre tecnologias ao ensino tradicional. A união de ambos os formatos potencializa a criação do conhecimento e, consequentemente, o aumento dos níveis de desempenho dos estudantes (Silva \& Bax, 2017).

Manter os alunos motivados consiste um dos pontos importantes na tarefa do docente. Todavia, essa motivação tornase aliada do processo de aprendizagem quando é intrínseca, ou seja, quando a iniciativa para aprender vem do aluno (Hanus; Fox, 2015). Nas dezesseis experiências da tabela acima os alunos demonstraram desejo próprio em se aprofundar nos conteúdos, o que evidencia que o objetivo da aplicação da gamificação foi atingido por parte dos docentes.

A gamificação pode ser entendida como um recurso adicional no trabalho do docente que visa motivar os alunos, além de proporcionar uma aprendizagem significativa. Todavia, para que as contribuições sejam efetivas, os objetivos de aprendizagem e o tipo de game precisam ser previamente definidos. Para tal, é necessário que os professores sejam capacitados para a utilização dessa estratégia de ensino e planejem os jogos de acordo com o nível de conhecimento dos estudantes e as competências almejadas (An, 2020).

\section{Considerações Finais}

Esta revisão de escopo serviu como estratégia oportuna para a realização de um mapeamento da literatura a respeito do tema trabalhado. Percebe-se que todos os estudos apontam benefícios no uso da gamificação como estratégia de ensino 
aprendizagem na formação do enfermeiro. Portanto, como mostra em um dos artigos, houve um ponto negativo em destaque: $\mathrm{o}$ estresse, pois os alunos se preocupavam em ficar atrasados nos games, se comparado aos seus pares.

Mesmo com as contribuições citadas neste estudo, destaca-se como limitação poucos estudos disponíveis na íntegra voltados ao curso de enfermagem que consolidem a efetividade desta metodologia. Sugere-se também a ampliação de futuras pesquisas referentes a temática, avaliando a eficácia da gamificação como estratégia de ensino e aprendizagem na formação do enfermeiro.

\section{Referências}

An, Y. (2020). Designing Effective Gamified Learning Experiences. International Journal of Technology in Education, 3(2), 62-69.

Arnab, S., \& Clarke, S. (2017). Towards a trans-disciplinary methodology for a game-based intervention development process. British journal of educational technology, 48(2), 279-312.

Araújo, I. (2016). Gamification: metodologia para envolver e motivar alunos no processo de aprendizagem. Educ Knowl Soc, 17(1), 87-107. https://www.researchgate.net/profile/Ines_Araujo4/publication/303463794_Gamification_metodologia_para_envolver_y_motivar_alumnos_en_el_proceso_de _aprendizaje/links/576838b708ae8ec97a4242ae.pdf.

Arksey, H., \& O'Malley, L. (2005). Scoping studies: towards a methodological framework. Int. j. soc. res. methodol. 8(1), 19-32.

Bellan, M. C., Alves, V. C., Neves, M. L. D. S., \& Lamas, J. L. T. (2017). Revalidação de jogo para ensino da medida auscultatória de pressão arterial: estudopiloto. Revista Brasileira de Enfermagem, 70(6), 1159-1168.

Castro, M. J., López, M., Cao, M. J., Fernández, C. M., García, S., Frutos, M., \& Jiménez, J. M. (2019). Impact of educational games on academic outcomes of students in the Degree in Nursing. PloS one, 14(7),1-5.

Conceição, C. V. D., \& Moraes, M. A. A. D. (2018). Aprendizagem Cooperativa e a Formação do Médico Inserido em Metodologias Ativas: um Olhar de Estudantes e Docentes. Rev. Bras. Educ. Med, 42(4), 115-122.

Chang, C. Y., Kao, C. H., Hwang, G. J., \& Lin, F. H. (2020). From experiencing to critical thinking: A contextual game-based learning approach to improving nursing students' performance in electrocardiogram training. Educational Technology Research and Development, 68(3), 1225-1245.

Chee, E. J. M., Prabhakaran, L., Neo, L. P., Carpio, G. A. C., Tan, A. J. Q., Lee, C. C. S., \& Liaw, S. Y. (2019). Play and Learn with Patients Designing and Evaluating a Serious Game to Enhance Nurses' Inhaler Teaching Techniques: A Randomized Controlled Trial. Games for health journal, 8(3), $187-194$.

Cruz, R. A. D. O., Araujo, E. L. M. D., Nascimento, N. D. M., Lima, R. J. D., França, J. R. F. D. S., \& Oliveira, J. D. S. (2017). Reflexões à luz da Teoria da Complexidade e a formação do enfermeiro. Rev. Bras. Enferm, 70(1), 236-239.

Da Silva Oliveira, G. K., Rodrigues, K. C., \& de Oliveira Freitas, M. A. (2018). Concepções de docentes sobre o uso de metodologias ativas da aprendizagem: um enfoque na formação de enfermeiros. Revista Sustinere, 6(2), 281-299.

Del Blanco, Á., Torrente, J., Fernández-Manjón, B., Ruiz, P., \& Giner, M. (2017). Using a videogame to facilitate nursing and medical students' first visit to the operating theatre. A randomized controlled trial. Nurse education today, 55(55), 45-53.

Fini, M.I., (2018). Inovações no ensino superior. Metodologias inovadoras de aprendizagem e suas relações com o mundo do trabalho: desafios para a transformação de uma cultura. Administração: Ensino e Pesquisa, 19(1), 176-183.

Giordano, C. V., \& Souza, L. T. D. (2021). A gamificação e a motivação dos alunos: considerações sobre técnicas efetivamente aplicadas na educação profissional. Revista Eniac Pesquisa, 10(1), 26-38.

Hanus, M. D., \& Fox, J. (2015). Assessing the effects of gamification in the classroom: A longitudinal study on intrinsic motivation, social comparison, satisfaction, effort, and academic performance. Computers \& Education, 80, 152-161.

Ignacio, J., \& Chen, H. C. (2020). The use of web-based classroom gaming to facilitate cognitive integration in undergraduate nursing students: A mixed methods study. Nurse Education in Practice, 46(46), 1-16.

Johnsen, H. M., Fossum, M., Vivekananda-Schmidt, P., Fruhling, A., \& Slettebo, A. (2018). Developing a serious game for nurse education. Journal of gerontological nursing, 44(1), 15-19.

Johnsen, H. M., Fossum, M., Vivekananda-Schmidt, P., Fruhling, A., \& Slettebo, A. (2018). Nursing students' perceptions of a video-based serious game's educational value: A pilot study. Nurse education today, 62(62), 62-68.

Johnsen, H. M., Briseid, H. S., Brodtkorb, K. Slettebo, A., \& Fossum, M. (2021). Nursing students' perceptions of combining hands-on simulation with simulated patients and a serious game in preparing for clinical placement in home healthcare: A qualitative study. Nurse education today, (97), 1-6.

Joseph, M. A., \& Natarajan, J. (2021). Muscle Anatomy Competition: Games Created by Nursing Students. Journal of Nursing Education, 60(4), $243-244$.

Mcenroe-petitte, D., \& Farris, C. (2020). O uso de jogos como estratégia ativa de ensino no ensino de enfermagem. Ensino e Aprendizagem em Enfermagem, $15(1), 61-65$. 
Michels, T. A., Paz, D. P., \& Ferreira, A. M. S. (2019). Gamificação como estratégia de ensino. Revista Mundi Engenharia, Tecnologia e Gestão, 4(1), 1-13.

Ministério da Saúde. (2018). Conselho Nacional de Saúde. Resolução Nº 573, de 31 de janeiro de 2018. Recomendações do Conselho Nacional de Saúde à proposta de Diretrizes Curriculares Nacionais (DCN) do curso de graduação Bacharelado em Enfermagem. Diário Oficial da União, 213 ed. Brasília, DF: Ministério da Saúde, 38.

Nascimento, J. L., \& Feitosa, R. A. (2020). Active methodologies, focusing on teaching and learning processes. Rev. Research, Society and Development, 9(9), $1-17$.

Orlandi, T. R., Duque, C. G., \& Mori, A. M. (2018). Gamificação: uma nova abordagem multimodal para a educação. Biblios, (70), 17-30.

Roman, P., Arrastia, M. R., Torres, G. M., Márquez-Hernández, V. V., Gutiérrez-Puertas, L., \& Ropero-Padilla, C. (2020). The escape room as evaluation method: A qualitative study of nursing students' experiences. Medical teacher, 42(4), 403-410.

Robson, K. (2019). Motivating professional student behavior through a gamified personal branding assignment. Journal of Marketing Education, 41(2), 154164.

Silva, A. N., Senna, M. A. A., Teixeira, C. B., Lucietto, D. A., \& Andrade, I. M. (2020). O uso de metodologia ativa no campo das Ciências Sociais em Saúde: relato de experiência de produção audiovisual por estudantes. Interface, (24), 1-14.

Silva, F. B., \& Bax, M. P. (2017). Gamification in online education: proposal for a participatory learning model. Encontros Bibli, 22(50), 144-160.

Silveira, M. S., \& Cogo, A. L. P. (2017). The contributions of digital technologies in the teaching of nursing skills: an integrative review. Rev Gaúcha Enferm., $38(2), 1-9$.

Solis de Ovando, A., Rodriguez, A., \& Hullin, C. (2018). Digital game-based learning: A didactic experience in the pre-degree nursing career. Nursing Informatics, (250), 88 .

Strickland, H. P., \& Kaylor, S. K. (2016). Bringing your a-game: Educational gaming for student success. Nurse Education Today, 40(40), 101-103.

Souza, E. F. D., Silva, A. G., \& Silva, A. I. L.F. (2018). Active methodologies for graduation in nursing: focus on the health care of older adults. Rev. Bras. Enferm. [Internet], (71), 976-980.

Tenório, T., Silva, A. R., \& Tenório, A. (2016). A influência da gamificação na Educação a Distância com base nas percepções de pesquisadores brasileiros. Revista EDaPECI São Cristóvão (SE). [Internet], 16(2), 320-335. http://www.seer.ufs.br/index.php/edapeci/article/view/4554/pdf.

Toledo, A. G. L., \& Kähler, P. Y. (2019). Empreendedorismo e inovação na gestão pública. Editora Inovar, 183.

Tricco, A. C. et al. (2018). Prisma Extension for Scoping Reviews (PRISMAScR): checklist and explanation. Ann Intern Med., 69(7), 467-473.

Verkuyl, M., Atack, L., Mastrilli, P., \& Romaniuk, D. (2016). Virtual gaming to develop students' pediatric nursing skills: A usability test. Nurse Education Today, 46 (46), 81-85.

Vieira, M. A. Souto, L. E. S., Souza, A. M., Lima, C. A., Ohara, C. V. S., \& Domenico, E. B. L. (2016). Diretrizes Curriculares Nacionais para a área da enfermagem: o papel das competências na formação do enfermeiro. Rev Norte Min Enferm [Internet], 5(1), $105-121$.

Yeo, C. L. et al. (2020). Use of web-based game in neonatal resuscitation-is it effective? BMC medical education, 20(1), 1-11. 